\title{
Linear ODE Based Geometric Modelling for Compressor Blades
}

\author{
Guanying Huo ${ }^{1,3}$, Xin Jiang ${ }^{1,3, *}$, Danlei $\mathrm{Ye}^{1,3}$, Cheng Su${ }^{1,3}$, Zehong $\mathrm{Lu}^{2}$, Bolun Wang ${ }^{1,3}$ and Zhiming Zheng ${ }^{1,3}$ \\ ${ }^{1}$ LMIB and School of Mathematics and Systems Science, Beihang University, Beijing, China \\ ${ }^{2}$ School of Mathematical Science, Peking University, Beijing, China \\ ${ }^{3}$ Big Data Brain Computing Center, Beihang University, Beijing, China \\ *Corresponding author
}

\begin{abstract}
Geometric modeling based on physical environment of the objects has been emerging as a powerful modeling technique and started to gain popularity for surface modeling and design. These methods usually allow the geometric objects to be defined and governed by a set of differential equations. In this paper, we focus on the geometric modeling for compressor blades, upon which the flow is governed by Navier-Stokes (NS) equations. As we cannot use NS equations directly due to its essential complexity, linear ODE systems are used to describe the elementary blade types of the blade surface. Then, we use the homotopy based method to reconstruct the full surface. A specific blade surface represented as data scatter is used to verify our method. According to the properties of the original data, different parametrization methods are discussed. Numerical results show that our method offers a nice representation for the blade surface.
\end{abstract}

Keywords-ODE systems; navier-stokes equations; homotopy

\section{INTRODUCTION}

Surface modeling techniques are vital to many visual computing fields such as interactive graphics, CAD/CAM, animation, and virtual environments [4]. A wide range of freeform splines have been developed during the last several decades [10-17]. However, traditional spline-based techniques can be difficult, time-consuming, less natural, and counterintuitive, primarily due to the reason that free-form splines are associated with tedious and indirect shape manipulation through time-consuming operations on a large number of (oftentimes irregular) control vertices, non-unity weights, and/or non-uniform knots. In addition, strong mathematical sophistication is necessary for users.

By contrast, modeling methods based on physical environment of the objects have recently started to gain popularity for surface modeling and design [1-9]. Compared with traditional control-point-based techniques, methods based on the physical environment have many advantages. Firstly, methods integrating physical laws are natural and much closer to the real world. Natural physical processes are frequently characterized by differential equations. Secondly, smooth surfaces with high-order continuity requirements can be readily defined through the use of complicated differential equations in modeling, which makes the following processes such as optimization and manufacture easier. Thirdly, users can easily understand the underlying physical processes associated with differential equations, therefore, intuitive and natural control is possible through the modification of physical parameters. At last, these methods can potentially unify both geometric and physical aspects. They are invaluable throughout the entire modeling, design, analysis, and manufacturing processes.

For describing natural physical processes, modeling methods based on PDEs are preferred when there is physical environment. However, we cannot get the analytical solutions of PDEs in most cases. Sometimes the numerical solutions of some equations are quite hard to achieve. PDE surfaces, as a typical one among these methods, allow geometric objects to be defined and governed by a set of elliptic or hyperbolic PDEs with different orders. They are easier to deal with, of which the solutions boast many good properties. However, these equations have no correlation with the original physical background. On the other hand, the forms of elliptic and hyperbolic PDEs are so special that they cannot describe all free-form surfaces. What is worse, even with such simple form, the properties of the solution cannot be achieved perfectly. Considering these shortcomings, we simplify the original PDEs in another way.

In this paper, we focus on the geometric modeling of compressor blades for aircraft. The movement of fluid upon the blades is governed by NS equation, of which the solution is proved to be quite complex. The proper shape of blade ensures good flow so that high efficiency and pressure ratio can be achieved. In turn, the corresponding flow make sense for shape design of the blade. We cannot use NS equation directly in modeling as we do not have an intuitive sense of what NS equations perform due to its complexity. Considering the importance of the flow upon the blade surface to the performance of compressor blade, we select ODE systems for shape representation as ODEs can easily generate flow with rich properties, different from PDE surfaces which are used for generating shapes. The solution curves of a set of ODEs give the description for the blade shapes. In this way, we can capture the properties of the middle part of the blade surface, other than PDE surfaces only operating on the boundaries of the elliptic or hyperbolic PDEs we use. Our work in this paper is to find out the corresponding ODEs for the original data. The original data we use are a set of elementary blade types obtained from the reverse engineering, which are given in the form of data scatter. Different parametrization methods are discussed to make the solution curves fit the original data better. After achieving the 
reconstruction of elementary blade types, we use the homotopy based method to complete the full surface. Finally, numerical results and some discussions of our method are given.

This paper is organized as follows. In Section 2, we give the ODEs based modeling method and discuss its mathematics fundamentals. In Section 3, the description of original data and numerical results are showed. Both qualitative and quantitative analysis are also given. We summarize our conclusion and give some discussion in the last section.

\section{GEOMETRIC MODELING FOR COMPRESSOR BLADES}

The compressor is used for compressing the air which flows through it to increase the efficiency of burning. The interaction between the shapes of compressor blades and the properties of the corresponding flow are discussed by engineers, especially designers, to provide better performance index of the compressor. The flow upon the blades is governed by NS equations, which we are not able to handle directly. In case of compressible and viscous fluid, the equations possess both hyperbolic and elliptic properties, which indicates the essential complexity of the equations. We try to simplify the NS equations. As one choice, ODE systems are familiar to most researchers because of their simplicity and practicality. In addition, linear ODE systems, as the simplest one among ODEs, are discussed thoroughly, which can also generate complex flow. The analytical solution of linear ODE can be achieved with the form $X(t)=e^{A t} X_{0}$ easily, making the analysis and adjustment for the shape more convenient. We prefer ODE systems over PDEs, as ODEs can generate field more intuitively. In this section, we introduce the ODE based shape representation for compressor blades, unifying both geometric and physical aspects. We argue this method can help us figure out the interaction between the shapes of compressor blades and the properties of the corresponding flow.

\section{A. Reconstruction of Elementary Blade Types}

In this subsection, we introduce the method to find out the corresponding linear ODE system for every elementary blade types. We formulate the ODE based shape representation method as following. Shape of the blade is given by data scatter of its corresponding elementary blade types, which are obtained from reverse engineering. We break every elementary blade types at the beginning and end points, which we call them suction line and pressure line respectively. The solution curves of two ODEs are used to fit one elementary blade type divided by the way stated. We try to find out the two ODE systems which approximate the elementary blade type under certain acceptable error, by which shape representation of the blade is given. Namely, assuming $\left\{X_{i}\right\}$ is the data scatter get through reverse engineer, we focus on finding out the linear ODEs whose solution curves fit the data well.

In the design process, the shape of blade is represent by a set of elementary blade types given in data scatter. Find out the linear ODEs to reconstruct the elementary blade types, then we can obtain the description for the shape of blade. We formulate the linear ODE as following:

$$
\left(\begin{array}{c}
\dot{x} \\
\dot{y} \\
\dot{z}
\end{array}\right)=\left(\begin{array}{lll}
a_{11} & a_{12} & a_{13} \\
a_{21} & a_{22} & a_{23} \\
a_{31} & a_{32} & a_{33}
\end{array}\right)\left(\begin{array}{l}
x \\
y \\
z
\end{array}\right)
$$

$x, y, z$ are the three-dimensional coordinates of one blade. Difference method is used here as we cannot handle the derivatives of $x, y, z$ directly. The simple difference scheme is applied,

$$
\left(\begin{array}{l}
\frac{x_{n+1}-x_{n}}{\Delta t_{n}} \\
\frac{y_{n+1}-y_{n}}{\Delta t_{n}} \\
\frac{z_{n+1}-z_{n}}{\Delta t_{n}}
\end{array}\right)=\left(\begin{array}{lll}
a_{11} & a_{12} & a_{13} \\
a_{21} & a_{22} & a_{23} \\
a_{31} & a_{32} & a_{33}
\end{array}\right)\left(\begin{array}{c}
x_{n} \\
y_{n} \\
z_{n}
\end{array}\right)
$$

$n$ represents the number of the original data. Let $A$ be the coefficient matrix, $X_{n}=\left(x_{n}, y_{n}, z_{n}\right)$. Achieving the two $A_{1}, A_{2}$, we can give the descriptions for both suction and pressure line of one elementary blade type, given the initial value of the ODEs and the corresponding $\Delta t_{n}$. To make the solution curves of the ODEs we find out closer to the original data, we need deal with the following unconstrained optimization problem,

$$
\min \sum_{n=1}^{N-1}\left\|\frac{\Delta X_{n}}{\Delta t_{n}}-A X_{n}\right\|
$$

The objective function evaluates the difference between the derivative value of the current point and the difference from the one to its adjacent point. Minimizing the objective function means we want to approximate the field generated by our ODE systems to the difference field of the original data. We use a series of notations to rearrange Eq. (2)

$$
D=\left(\begin{array}{ccc}
d_{1}\left(=\frac{x_{2}-x_{1}}{\Delta t_{1}}\right) & \cdots & d_{n-1} \\
e_{1}\left(=\frac{y_{2}-y_{1}}{\Delta t_{1}}\right) & \cdots & e_{n-1} \\
f_{1}\left(=\frac{z_{2}-z_{1}}{\Delta t_{1}}\right) & \cdots & f_{n-1}
\end{array}\right)
$$




$$
\begin{aligned}
& \alpha_{1}=\left(x_{1}, \cdots, x_{n-1}\right), \alpha_{2}=\left(y_{1}, \cdots, y_{n-1}\right), \alpha_{3}=\left(z_{1}, \cdots, z_{n-1}\right) \\
& \beta_{1}=\left(d_{1}, \cdots, d_{n-1}\right), \beta_{2}=\left(e_{1}, \cdots, e_{n-1}\right), \beta_{3}=\left(f_{1}, \cdots, f_{n-1}\right)
\end{aligned}
$$

Then, Eq. (3) can be expanded

$$
\begin{aligned}
\left.\min _{A} \sum_{n=1}^{N-1}+\left(a_{11} x_{n}+a_{12} y_{n}+a_{13} z_{n}-d_{n}\right)^{2}+\left(a_{32} x_{n}+a_{22} y_{n} y_{33} z_{n}-a_{23} z_{n}\right)^{2}\right] \\
\min _{\left\{a_{1 i}\right\}} \sum_{n=1}^{N-1}\left(a_{11} x_{n}+a_{12} y_{n}+a_{13} z_{n}-d_{n}\right)^{2}+ \\
\min _{\left\{a_{2 i}\right\}} \sum_{n=1}^{N-1}\left(a_{21} x_{n}+a_{22} y_{n}+a_{23} z_{n}-e_{n}\right)^{2}+ \\
\min _{\left\{a_{3 i}\right\}} \sum_{n=1}^{N-1}\left(a_{31} x_{n}+a_{32} y_{n}+a_{33} z_{n}-f_{n}\right)^{2}
\end{aligned}
$$

Take $\min _{\left\{a_{1 i}\right\}} \sum_{n=1}^{N-1}\left(a_{11} x_{n}+a_{12} y_{n}+a_{13} z_{n}-d_{n}\right)^{2}, i=1, \cdots, 3$ as example. According to the method of Lagrange multipliers, we have

$$
\begin{aligned}
& \left(a_{11} \alpha_{1}+a_{12} \alpha_{2}+a_{13} \alpha_{3}-\beta_{1}\right) \alpha_{i}^{T}=0, i=1, \cdots, 3 \\
& \left(a_{11} \alpha_{1}+a_{12} \alpha_{2}+a_{13} \alpha_{3}-\beta_{1}\right) X^{T}=0 \\
& \left(\left(a_{11}, a_{12}, a_{13}\right) X-\beta_{1}\right) X^{T}=0 \\
& \left(a_{11}, a_{12}, a_{13}\right) X X^{T}=\beta_{1} X^{T} \\
& \left(a_{11}, a_{12}, a_{13}\right)=\beta_{1} X^{T}\left(X X^{T}\right)^{-1}
\end{aligned}
$$

We can also get

$$
\begin{aligned}
& \left(a_{11}, a_{12}, a_{13}\right)=\beta_{1} X^{T}\left(X X^{T}\right)^{-1} \\
& \left(a_{21}, a_{22}, a_{23}\right)=\beta_{2} X^{T}\left(X X^{T}\right)^{-1} \\
& \left(a_{31}, a_{32}, a_{33}\right)=\beta_{3} X^{T}\left(X X^{T}\right)^{-1}
\end{aligned}
$$

Then, $A$ is achieved by $A=D X^{T}\left(X X^{T}\right)^{-1} .\left\{\Delta t_{n}\right\}$ is set according to parametrization method of the original data. We achieve different results with different sets of $\left\{\Delta t_{n}\right\}$.

It is a least square method. Our goal is to make the derivative of solution curves close to the difference between adjacent data at the given point of original scatter. If the adjacent data are given densely enough, the original scatter can be treated as on the solution curves we get. From a deeper perspective, we try to find out the best fitted flow to the original data.

\section{B. Reconstruction of Blade Surface}

We use the linear ODE systems to represent the elementary blade types in last subsection. The solution curves of the ODEs can be treated as the skeleton of the blade surface. In traditional ways, designers use the interpolation methods to fill the rest. However, these methods can only provide discrete description, which makes the following optimization and manufacture difficult. In this subsection, we provide a new method to achieve this, with the help of homotopy theory.

Blades with high aerodynamic performance need smooth surfaces. Achieving a set of ODE systems to describe the suction and pressure lines of elementary blade types, we try to transfer one system to the adjacent one naturally to fill the rest between them. A method based on homotopy theory is proposed.

As we described above, the blade surface is treated as the flow governed by ODE systems we get. Given the start side of the rest of the blade surface, we try to find out the corresponding systems, with which the full surface can be achieved. Firstly, we represent the start point of the system to be generated as the linear combination of the start points of the two adjacent systems among the skeleton, namely, $X_{0 u}=u X_{0 i}+(1-u) X_{0(i+1)}, 0 \leq u \leq 1 . X_{0 u}$ is between $X_{0 i}$ and $X_{0(i+1)}$. Repeat the process to the rest data with the same parameter $u$ of the two adjacent systems, then a new data for the system to be generated is obtained. We can use the method proposed in Section 2.1 to achieve the system we need. Given a parameter $u$, we can find out the corresponding system in this way. The surface we achieve can be as smooth as we need with proper $\Delta u$. The geometric modeling of the blade is finished. We formulate the process as follows $X_{j u}=u X_{j i}+(1-u) X_{j(i+1)}, 0 \leq u \leq 1,0 \leq j \leq K, K$ is the number of points in the suction and pressure line. Then $A(u)=D(u) X(u)^{T}\left(X(u) X(u)^{T}\right)^{-1}$, where $D(u)$ means replacing the $X$ by $X(u)$ in its computation (Eq. (4)).

We use ODE systems to reconstruct the suction and pressure lines of elementary blade types. Homotopy based method is introduced to complete the rest surface. Finally, the shape of the blade is described with the solution curves of a set of ODEs. A new geometric model for compressor blade is given, with which we can easily integrate the following processes such as optimization and manufacture.

The overall algorithm for reconstructing the shape of compressor blade is summarized:

Step.1 Accept the original data, and break them into suction and pressure lines.

Step.2 Parameterize the scattered points of suction and pressure lines.

Step.3 Solve the coefficients A of the linear ODEs through the least square method.

Step.4 Find out the corresponding ODEs for the rest part of the blade surface as densely as we need. 
Step.5 Solve all the linear ODEs we get, the solution curve is finally described with the form $l(u, t)=e^{A(u) t} X_{0 u}$.

\section{RESUlTS AND DisCUSSIONS}

The input of the proposed algorithm are the 3D data scatter of a specific blade (see Figure 1). The data we used contains 15 elementary blade types, which can be divided into 15 suction and pressure lines respectively. Each line has 71 points. Our algorithm tries to find out the corresponding ODE systems, whose solution curves fit the data well.

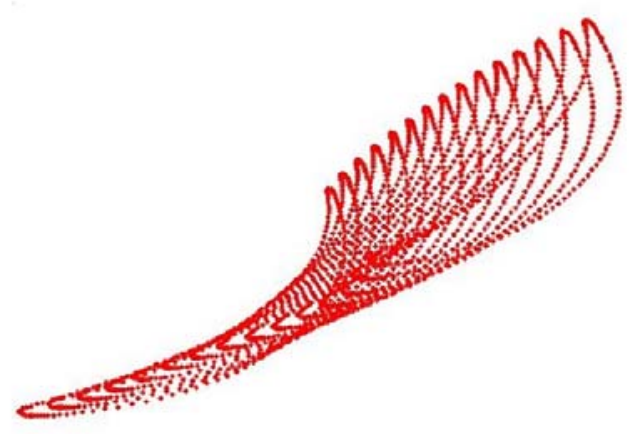

FIGURE I. THE DATA SCATTER OF THE ORIGINAL BLADE

The code was developed in Matlab on a PC with a 3.33 $\mathrm{GHz}$ Intel(R) I5 processor and 4.0 GB RAM. Before the least square procedure, we need parameterize the original data. The accumulative chord length parametrization is used because of its homogeneity. Namely, we set

$$
\Delta t_{n}=\frac{\left\|\Delta X_{n}\right\|}{\sum_{n=1}^{N-1}\left\|\Delta X_{n}\right\|}, \Delta X_{n}=X_{n+1}-X_{n}
$$

\section{A. Qualitative Results}

Numerical result of our algorithm is showed in Figure 2, compared with uniform parametrization $\Delta t_{n} \equiv 1$. To make the results clearer, we only show the simulation of suction lines.

$\Delta t_{n} \equiv 1$ means the step size of the adjacent points of original data is the same. Namely, a point moves to his next point with the same time. The black lines in Fig. 2 give the numerical result of this condition. The curves given by our ODEs do not satisfy the data well at some points. Checking the original data, we find that the distances between adjacent points are asymmetry, which leads to the asymmetry distribution of the velocity field. The flow generated by our ODE is hard to answer for this. By contrast, accumulative chord length parametrization is used, which indicates the well-distribution flow. In this case, we assume that the velocity of fluid changes little during one suction or pressure line. The blue lines in Figure 2 show the corresponding results. We can see that welldistributed flow fits the original data better.

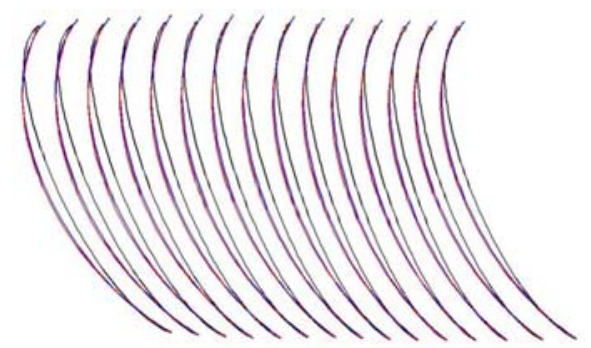

FIGURE II. THE NUMERICAL RESULT OF SUCTION LINES USING OUR METHOD WITH DIFFERENT TIME STEPS. THE RED LINES ARE THE ORIGINAL DATA, AND THE BLACK LINES ARE WHAT WE GET WITH ASYMMETRY-DISTRIBUTION FLOW FIELD, WHILE THE BLUE ONES GET WITH WELL-DISTRIBUTE FLOW FIELD

We argue that the linear ODEs is enough to reconstruct the suction and pressure lines, although they are very simple. Capturing the essence of the flow, the linear ODE systems offer a proper representation for the shape of elementary blade types of compressor blades, unifying both geometric and physical aspects. We can solve the linear ODEs easily using Runge-Kutta method with sufficient precision and negligible time in our computer as their forms are much easier than other spline-based methods such as NUBRS. On the other hand, the surface we achieve can be expressed analytically, i.e. $l(u, t)=e^{A(u) t} X_{0 u}$ for fixed parameter $u$, boasting infinite order of smooth derivative, which means a lot for shape designers. Interpolation condition across curves in the end can be satisfied by introducing second order linear ODEs with the form $\ddot{X}=A_{1} \dot{X}+A_{2} X$. The process is the same.

Achieving the corresponding linear ODE systems for suction and pressure lines of elementary blade types, we use the homotopy based method introduced in Section 2.2 to fill the rest of the blade surface. Numerical results are given in Figure 3. The homotopy based method gives a proper description for the full blade surface with good smoothness. In fact, the final description of the full blade surface can be expressed analytically as $S(u, t)=e^{A(u) t} l(u)$ if we use a continuous curve to describe the starting point of the blade surface, which boasts infinite order of smooth partial derivative. In addition, we can easily adjust the surface in this form in the following optimization and manufacture processes due to the simplicity and integrity.

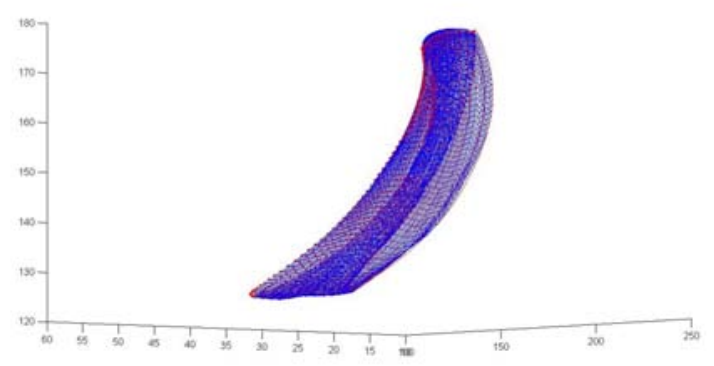

FIGURE III. THE NUMERICAL RESULT OF THE BLADE SURFACE. THE RED LINE IS THE ORIGINAL DATA, AND THE BLUE LINE IS WHAT WE ACHIEVE THROUGH OUR METHOD 


\section{B. Quantitative Results}

In the last subsection, we consider various metrics to analyze our algorithm qualitatively. Quantitative results comparing solution curves of ODEs we obtain with original data will be shown in this subsection, to test our algorithm further.

The blade we used as the original data is about $100 \mathrm{~mm}$ in length. We apply the accumulative chord length parametrization. The fourth order Runge-Kutta method is used to compute the solution curves of the ODEs we achieve. We define the absolute errors as the absolute value of the difference between the original data and the corresponding points of the solution curve. The pair of points have the same parameter values. There are 15 suction lines, and every line has 73 points. Define the maximum absolute value as the maximum of the 73 absolute errors of every suction line. We plot the 15 errors in Fig.4. In addition, we define the maximum relative error as the maximum absolute error divided by the coordinates of the points in the suction lines. The results are showed in Fig.5.

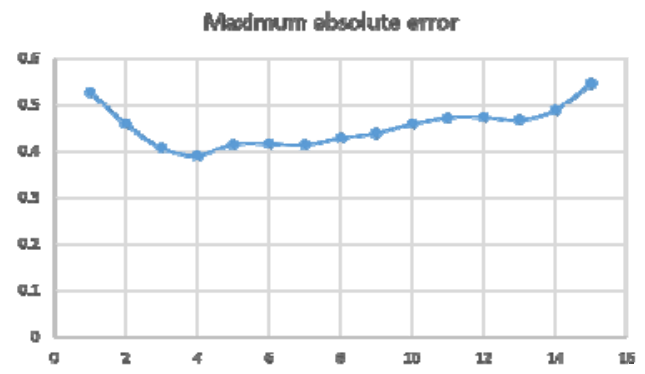

FIGURE IV. THE MAXIMUM ABSOLUTE ERRORS OF THE 15 SUCTION LINES

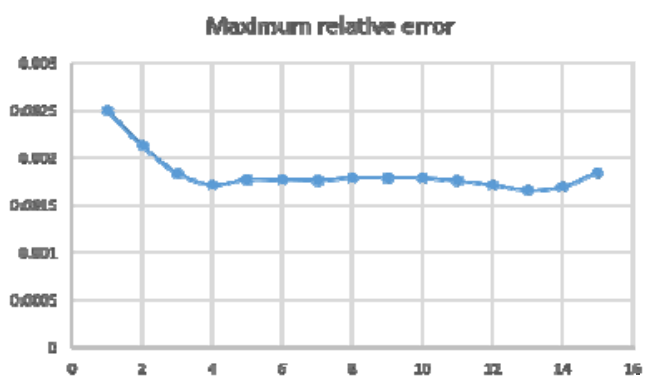

FIGURE V. THE MAXIMUM RELATIVE ERRORS OF THE 15 SUCTION LINES

The quantitative results demonstrate the superiority of our method. With the simple linear ODE systems, we can represent the complex suction line of blade successfully, which itself is a curve with strong three-dimensional characteristics. Moreover, our method is easier to compute than other spline-based methods due to its simplicity. We can use nonlinear ODEs and segmental technology to improve the precision of our method if stricter requirement for precision is to meet.

\section{CONCLUSION}

We have investigated a new geometric modeling method for compressor blade using linear ODE systems. The data of a specific blade is used for our simulations. Considering properties of the original data scatter, we discuss different parametrization methods. Numerical results show that linear ODEs can represent both the suction and pressure lines of elementary blade types well, and homotopy based method can reconstruct the full surface with satisfied smoothness. Qualitative and quantitative analysis are conducted to evaluate the performance of the proposed algorithm further. Our future work may focus on the elementary surface types that our method can generate. More complicated ODEs can also be used. Further, the application of the blade surface represented in our form to the following optimization and manufacture processes will be studied.

\section{ACKNOWLEDGMENT}

This work is partially supported by the Chinese National Science Foundation under Grant No.11290141, No.11571028.

\section{REFERENCES}

[1] M. I. G. Bloor, M. J. Wilson. Generating blend surfaces using partial differential equations[J]. Computer-Aided Design, 1989, 21(3): 165-171.

[2] M. I. G. Bloor, M. J. Wilson. Using partial differential equations to generate free-form surfaces[J]. Computer-Aided Design, 1990, 22(4): 202-212.

[3] H. Ugail, M. I. G. Bloor, M. J. Wilson. Techniques for interactive design using the PDE method[J]. ACM Transactions on Graphics (TOG), 1999, 18(2): 195-212.

[4] H. Du, H. Qin. Dynamic PDE surfaces with flexible and general geometric constraints[C]//Computer Graphics and Applications, 2000. Proceedings. The Eighth Pacific Conference on. IEEE, 2000: 213-447.

[5] G. G. Castro, H. Ugail, P. Willis, et al. A survey of partial differential equations in geometric design[J]. The Visual Computer, 2008, 24(3): 213-225.

[6] S. P. Lim, H. Haron. Surface reconstruction techniques: a review[J]. Artificial Intelligence Review, 2014, 42(1): 59-78.

[7] G. Xu, Q. Pan, C. L. Bajaj. Discrete surface modelling using partial differential equations[J]. Computer Aided Geometric Design, 2006, 23(2): 125-145.

[8] Y. Suzuki, K. T. Miura, I. Tanaka, et al. Streamline modeling based on potential flow[C]. Computer Graphics and Applications, 2001. Proceedings. Ninth Pacific Conference on. IEEE, 2001: 305-312.

[9] X. Lu. Construct streamline surface by restricted Bézier patch[C]. Computer and Information Technology, 2004. CIT'04. The Fourth International Conference on. IEEE, 2004: 759-763.

[10] G. Xu, Q. Zhang. A general framework for surface modeling using geometric partial differential equations[J]. Computer Aided Geometric Design, 2008, 25(3): 181-202.

[11] C. De Boor. A practical guide to splines[J]. 1978.

[12] G. E. Farin. Curves and surfaces for computer-aided geometric design: a practical code[M]. Academic Press, Inc., 1996.

[13] L. Piegl. On NURBS: a survey[J]. IEEE Computer Graphics and Applications, 1991, 11(1): 55-71.

[14] L. Piegl, W. Tiller. Curve and surface constructions using rational Bsplines[J]. Computer-Aided Design, 1987, 19(9): 485-498.

[15] D. Saini, S. Kumar, T. R. Gulati. Reconstruction of free-form space curves using NURBS-snakes and a quadratic programming approach[J]. Computer Aided Geometric Design, 2015.

[16] G. Maier. Optimal arc spline approximation[J]. Computer Aided Geometric Design, 2014, 31(5): 211-226.

[17] Y. C. Kim, T. W. Kim, S. Pyo, et al. Design of propeller geometry using streamline-adapted blade sections[J]. Journal of marine science and technology, 2009, 14(2): 161-170. 